\title{
COMPARISON OF DIFFERENTIAL REPRESENTATIONS FOR RADIALLY SYMMETRIC STOKES FLOW
}

\author{
GEORGE DASSIOS AND PANAYIOTIS VAFEAS
}

Received 10 September 2002

Papkovich and Neuber (PN), and Palaniappan, Nigam, Amaranath, and Usha (PNAU) proposed two different representations of the velocity and the pressure fields in Stokes flow, in terms of harmonic and biharmonic functions, which form a practical tool for many important physical applications. One is the particle-in-cell model for Stokes flow through a swarm of particles. Most of the analytical models in this realm consider spherical particles since for many interior and exterior flow problems involving small particles, spherical geometry provides a very good approximation. In the interest of producing ready-to-use basic functions for Stokes flow, we calculate the PNAU and the PN eigensolutions generated by the appropriate eigenfunctions, and the full series expansion is provided. We obtain connection formulae by which we can transform any solution of the Stokes system from the PN to the PNAU eigenform. This procedure shows that any PNAU eigenform corresponds to a combination of PN eigenfunctions, a fact that reflects the flexibility of the second representation. Hence, the advantage of the PN representation as it compares to the PNAU solution is obvious. An application is included, which solves the problem of the flow in a fluid cell filling the space between two concentric spherical surfaces with Kuwabara-type boundary conditions.

\section{Introduction}

Slow motion of a mass of particles relative to a viscous fluid has been studied extensively because of its importance in practical applications. In order to construct tractable mathematical models of the flow systems involving particles, it is necessary to conform to a number of simplifications. A dimensionless criterion, which determines the relative importance of inertial and viscous effects, is the Reynolds number [3]. Stokes equations for the steady flow of a viscous, incompressible fluid at small Reynolds number (creeping flow) have been known for over one and a half centuries (1851). They connect the vector velocity with the scalar total pressure field [3]. The total pressure and vorticity fields are harmonic, while the velocity is biharmonic and divergence-free. 
Complications often arise because of the complex geometry encountered in assemblages composed of particles of arbitrary shape. There are many efficient methods in use to solve this kind of problems with Stokes flow, such as numerical computation, streamfunction techniques, and analytic-function methods [10]. One of the largest physical areas of importance concerns the construction of particle-in-cell models which are useful in the development of simple but reliable analytical expressions for heat and mass transfer in swarms of particles. The technique of cell models is based on the idea according to which a large enough porous concentration of particles within a fluid can be represented by many separate unit cells where every cell contains one particle. Thus, the consideration of a full-dimensional porous media is being referred to as that of a single particle and its fluid cover. That way, the mathematical formulation of any physical problem is significantly simplified. For many interior and exterior flow problems involving small particles, spherical geometry [6] provides very good approximation and stands for the simplest geometry that can be employed. Although relative physical problems enjoy rotational symmetry, we retain the nonaxisymmetric character of three-dimensional (3D) flows.

The introduction of differential representations of the solutions of Stokes equations $[1,8,9,10]$ serves to unify our own approach on all $3 \mathrm{D}$ incompressible fluid motions. Based on the previous formulation of cell models, the problem is now focused on the use of the appropriate representation that coincides with the physical problem. The major advantage of the differential representations is that they provide us with the flow fields for Stokes flow in terms of harmonic potentials. The most famous general spatial solutions are the PN solution $[8,10]$, the Boussinesq-Galerkin solution $[1,10]$, and the PNAU solution [9]. Recently, a method of connecting 3D differential representations has been developed [2], where the PN and the Boussinesq-Galerkin differential representations were interrelated and connection formulae between the corresponding spherical harmonic and biharmonic potentials were developed.

Here we are interested in the connection of the PN solution with the PNAU representation in spherical coordinates. This is made possible by connecting the appropriate eigenfunctions that generate the flow fields through these representations. Our aim is to calculate the nonaxisymmetric flow fields, generated by the vector spherical harmonic eigenfunctions $[4,7]$, through the PN representation and then to face the inverse problem of determining those vector spherical harmonic and biharmonic eigenfunctions $[4,6,10]$, which lead to the same velocity and total pressure fields via the PNAU representation. Furthermore, both the internal and the external flow problems are being examined. The above procedure cannot be inverted as a consequence of the flexibility that the PN representation enjoys as it compares to the PNAU solution. This indicates that the use of the $\mathrm{PN}$ differential representation forms a more complete way to solve particle-in-cell flow problems.

As a demonstration of the usefulness and the possibilities offered by the PN representation, we derive the solution of the problem of creeping flow through a swarm of stationary spherical particles, embedded within an otherwise quiescent Newtonian fluid that moves with constant uniform velocity in the axial direction using the Kuwabara-type boundary conditions [5]. 


\section{Fundamentals of stokes flow}

Stokes flow which is characterized by steady, nonaxisymmetric $3 \mathrm{D}$, creeping $(\operatorname{Re} \ll 1)$, incompressible (density $\varrho=$ const), and viscous (dynamic viscosity $\mu=$ const) motion around particles embedded within smooth, bounded domains $\Omega\left(\mathbb{R}^{3}\right)$ is governed by the following set of partial differential equations [3]:

$$
\begin{gathered}
\mu \Delta \mathbf{v}(\mathbf{r})-\nabla \mathrm{P}(\mathbf{r})=\mathbf{0}, \quad \mathbf{r} \in \Omega\left(\mathbb{R}^{3}\right), \\
\nabla \cdot \mathbf{v}(\mathbf{r})=0, \quad \mathbf{r} \in \Omega\left(\mathbb{R}^{3}\right),
\end{gathered}
$$

where $\mathbf{v}(\mathbf{r})$ is the biharmonic velocity field, $\mathrm{P}(\mathbf{r})$ is the harmonic total pressure field, and $\mathbf{r}$ stands for the position vector. An immediate consequence of (2.1) is that, for creeping flow, the generated pressure is compensated by the viscous forces while equation (2.2) secures the incompressibility of the fluid. Once the velocity field is obtained, the harmonic vorticity field is defined as

$$
\boldsymbol{\omega}(\mathbf{r})=\nabla \times \mathbf{v}(\mathbf{r}), \quad \mathbf{r} \in \Omega\left(\mathbb{R}^{3}\right) .
$$

Papkovich and Neuber [8] proposed the following 3D differential representation of the solutions for Stokes flow, in terms of the harmonic potentials $\Phi(\mathbf{r})$ and $\Phi_{0}(\mathbf{r})$ :

$$
\begin{aligned}
& \mathbf{v}^{\mathrm{PN}}(\mathbf{r})=\boldsymbol{\Phi}(\mathbf{r})-\frac{1}{2} \nabla\left(\mathbf{r} \cdot \boldsymbol{\Phi}(\mathbf{r})+\Phi_{0}(\mathbf{r})\right), \quad \mathbf{r} \in \Omega\left(\mathbb{R}^{3}\right), \\
& \mathrm{P}^{\mathrm{PN}}(\mathbf{r})=\mathrm{P}_{0}^{\mathrm{PN}}-\mu \nabla \cdot \boldsymbol{\Phi}(\mathbf{r}), \quad \mathbf{r} \in \Omega\left(\mathbb{R}^{3}\right),
\end{aligned}
$$

whereas $\mathrm{P}_{0}^{\mathrm{PN}}$ is a constant pressure and

$$
\Delta \Phi(\mathbf{r})=\mathbf{0}, \quad \Delta \Phi_{0}(\mathbf{r})=0, \quad \mathbf{r} \in \Omega\left(\mathbb{R}^{3}\right) .
$$

On the other hand, Palaniappan et al. [9] assumed another 3D differential representation for the solutions of Stokes equations as a function of the harmonic and biharmonic potentials $\mathrm{A}(\mathbf{r})$ and $\mathrm{B}(\mathbf{r})$, respectively:

$$
\begin{aligned}
& \mathbf{v}^{\mathrm{PNAU}}(\mathbf{r})=\nabla \times \nabla \times(\mathbf{r A}(\mathbf{r}))+\nabla \times(\mathbf{r B}(\mathbf{r})), \quad \mathbf{r} \in \Omega\left(\mathbb{R}^{3}\right), \\
& \mathrm{P}^{\mathrm{PNAU}}(\mathbf{r})=\mathrm{P}_{0}^{\mathrm{PNAU}}+\mu(1+\mathbf{r} \cdot \nabla) \Delta \mathrm{A}(\mathbf{r}), \quad \mathbf{r} \in \Omega\left(\mathbb{R}^{3}\right),
\end{aligned}
$$

where $\mathrm{P}_{0}^{\mathrm{PNAU}}$ is a constant pressure, while

$$
\Delta^{2} \mathrm{~A}(\mathbf{r})=0, \quad \Delta \mathrm{B}(\mathbf{r})=0, \quad \mathbf{r} \in \Omega\left(\mathbb{R}^{3}\right),
$$

and $\Delta$ and $\nabla$ stand for the Laplacian and the gradient operators, respectively.

In what follows, we find the interrelation of these differential representations in order to obtain connection formulae between the spherical harmonic $\left(\boldsymbol{\Phi}, \Phi_{0}, \mathrm{~B}\right)$ and biharmonic (A) eigenfunctions. Putting it in a different way, given an eigenmode of one of the representations, we look for the particular combination of eigenmodes of the other representation that generates the same velocity and total pressure fields. Initially, the physically 
important internal and external fields of the velocity and the total pressure $(\mathbf{v}, \mathrm{P})$ are constructed using the representations (2.4) and (2.6). Since spherical geometry is employed, we are using vector spherical harmonics [7] in order to simplify our calculations.

Furthermore, in order to demonstrate the usefulness of the PN differential representation, we use it to solve the Stokes flow problem within a fluid cell limited between two concentric spherical surfaces. In this way, we are led to recover the solution of the Kuwabara-type problem [5] for the small Reynolds number flow around spheres embedded in a viscous fluid.

\section{Vector spherical harmonic and biharmonic eigenfunctions}

Introducing the spherical coordinate system $[6](\zeta=\cos \theta,-1 \leq \zeta \leq 1)$,

$$
x_{1}=r \sqrt{1-\zeta^{2}} \cos \varphi, \quad x_{2}=r \sqrt{1-\zeta^{2}} \sin \varphi, \quad x_{3}=r \zeta,
$$

where $0 \leq r<+\infty, 0 \leq \theta \leq \pi$, and $0 \leq \varphi<2 \pi$, we define the sphere $B_{r}$ for $r>0$ as the set

$$
B_{r}=\left\{\mathbf{r} \in \mathbb{R}^{3} \mid x_{1}^{2}+x_{2}^{2}+x_{3}^{2} \leq r^{2}\right\} .
$$

The outward unit normal vector on the surface of the sphere $r=r_{0}$ is furnished by the formula

$$
\hat{\mathbf{n}}\left(r_{0}, \zeta, \varphi\right)=\left(\sqrt{1-\zeta^{2}} \cos \varphi, \sqrt{1-\zeta^{2}} \sin \varphi, \zeta\right)=\frac{\mathbf{r}\left(r_{0}, \zeta, \varphi\right)}{r_{0}},
$$

where for any nondegenerate sphere $B_{r_{0}}$, we have $r_{0}>0$. Furthermore, $|\zeta| \leq 1$. The differential operators $\nabla$ and $\Delta$, in spherical coordinates, assume the forms

$$
\begin{aligned}
& \nabla=\hat{\mathbf{r}} \frac{\partial}{\partial r}-\frac{\sqrt{1-\zeta^{2}}}{r} \hat{\zeta} \frac{\partial}{\partial \zeta}+\frac{1}{r \sqrt{1-\zeta^{2}}} \hat{\boldsymbol{\varphi}} \frac{\partial}{\partial \varphi}, \\
& \Delta=\frac{1}{r^{2}} \frac{\partial}{\partial r}\left(r^{2} \frac{\partial}{\partial r}\right)+\frac{1}{r^{2}} \frac{\partial}{\partial \zeta}\left[\left(1-\zeta^{2}\right) \frac{\partial}{\partial \zeta}\right]+\frac{1}{r^{2}\left(1-\zeta^{2}\right)} \frac{\partial^{2}}{\partial \varphi^{2}},
\end{aligned}
$$

while $\hat{\mathbf{r}}, \hat{\zeta}$, and $\hat{\boldsymbol{\varphi}}$ stand for the coordinate unit vectors of our system for $r>0$ and $|\zeta| \leq 1$.

For every value of $n=0,1,2, \ldots$, there exist $(2 n+1)$ linearly independent spherical surface harmonics [4] given by

$$
Y_{n}^{m s}(\hat{\mathbf{r}})=P_{n}^{m}(\zeta) \begin{cases}\cos m \varphi, & s=e, \\ \sin m \varphi, & s=o,\end{cases}
$$

for $m=0,1,2, \ldots, n,|\zeta| \leq 1, \varphi \in[0,2 \pi)$, where

$$
\oint_{S^{2}} Y_{n}^{m s}(\hat{\mathbf{r}}) Y_{n^{\prime}}^{m^{\prime} s^{\prime}}(\hat{\mathbf{r}}) d S(\hat{\mathbf{r}})=\frac{4 \pi}{2 n+1} \frac{(n+m) !}{(n-m) !} \delta_{n n^{\prime}} \delta_{m m^{\prime}} \delta_{s s^{\prime}} \frac{1}{\varepsilon_{m}},
$$

with $\delta_{i j}, i=n, m, s, j=n^{\prime}, m^{\prime}, s^{\prime}$, the Kronecker delta, $\varepsilon_{m}$ the Neumann factor $\left(\varepsilon_{m}=1\right.$, $m=0$, and $\varepsilon_{m}=2, m \geq 1$ ), and $s$ denoting the even (e) or the odd (o) character of the 
spherical surface harmonics; $P_{n}^{m}=P_{n}^{m}(\zeta)$ are the associated Legendre functions of the first kind [4] given by the relation

$$
P_{n}^{m}(\zeta)=\frac{\left(1-\zeta^{2}\right)^{m / 2}}{2^{n} n !} \frac{d^{n+m}}{d \zeta^{n+m}}\left(\zeta^{2}-1\right)^{n}, \quad|\zeta|<1,
$$

where $n$ denotes the degree and $m$ the order.

In spherical coordinates, the linear space of harmonic functions can be expressed via the complete set of internal and external solid spherical harmonics, that is,

$$
\Delta g(\mathbf{r})=0 \Longleftrightarrow g(\mathbf{r})=\left\{\begin{array}{l}
r^{n} Y_{n}^{m s}(\hat{\mathbf{r}}), \\
r^{-(n+1)} Y_{n}^{m s}(\hat{\mathbf{r}}),
\end{array}\right.
$$

for $n \geq 0, m=0,1, \ldots, n$, and $s=e, o$. Similarly, according to the representation theorem of Almansi (1897) [10], every biharmonic function permits an appropriate decomposition into two harmonic functions $h_{1}(\mathbf{r})$ and $h_{2}(\mathbf{r})$, that is,

$$
h(\mathbf{r})=h_{1}(\mathbf{r})+r^{2} h_{2}(\mathbf{r}) \quad \text { with } \Delta h_{1}(\mathbf{r})=\Delta h_{2}(\mathbf{r})=0 .
$$

For every $-1 \leq \zeta \leq 1$ and $\varphi \in[0,2 \pi)$, the vector spherical surface harmonics [7] which are defined by the relations

$$
\begin{aligned}
& \mathbf{P}_{n}^{m s}(\hat{\mathbf{r}})=\hat{\mathbf{r}} Y_{n}^{m s}(\hat{\mathbf{r}}), \\
& \mathbf{B}_{n}^{m s}(\hat{\mathbf{r}})=\frac{1}{\sqrt{n(n+1)}}\left[-\sqrt{1-\zeta^{2}} \hat{\zeta} \frac{\partial}{\partial \zeta}+\frac{1}{\sqrt{1-\zeta^{2}}} \hat{\boldsymbol{\varphi}} \frac{\partial}{\partial \varphi}\right] Y_{n}^{m s}(\hat{\mathbf{r}}), \\
& \mathbf{C}_{n}^{m s}(\hat{\mathbf{r}})=-\frac{1}{\sqrt{n(n+1)}} \hat{\mathbf{r}} \times\left[-\sqrt{1-\zeta^{2}} \hat{\zeta} \frac{\partial}{\partial \zeta}+\frac{1}{\sqrt{1-\zeta^{2}}} \hat{\boldsymbol{\varphi}} \frac{\partial}{\partial \varphi}\right] Y_{n}^{m s}(\hat{\mathbf{r}}),
\end{aligned}
$$

for any $n \geq 0, m=0,1, \ldots, n$, and $s=e, o$, are pointwise perpendicular; that is,

$$
\mathbf{P}_{n}^{m s} \cdot \mathbf{C}_{n}^{m s}=\mathbf{C}_{n}^{m s} \cdot \mathbf{B}_{n}^{m s}=\mathbf{B}_{n}^{m s} \cdot \mathbf{P}_{n}^{m s}=0
$$

Moreover they satisfy the orthogonality relations

$$
\begin{aligned}
\oint_{S^{2}} \mathbf{P}_{n}^{m s}(\hat{\mathbf{r}}) \cdot \mathbf{P}_{n^{\prime}}^{m^{\prime} s^{\prime}}(\hat{\mathbf{r}}) d S(\hat{\mathbf{r}}) & =\oint_{S^{2}} \mathbf{B}_{n}^{m s}(\hat{\mathbf{r}}) \cdot \mathbf{B}_{n^{\prime}}^{m^{\prime} s^{\prime}}(\hat{\mathbf{r}}) d S(\hat{\mathbf{r}}) \\
& =\oint_{S^{2}} \mathbf{C}_{n}^{m s}(\hat{\mathbf{r}}) \cdot \mathbf{C}_{n^{\prime}}^{m^{\prime} s^{\prime}}(\hat{\mathbf{r}}) d S(\hat{\mathbf{r}}) \\
& =\frac{4 \pi}{2 n+1} \frac{(n+m)}{(n-m)} \delta_{n n^{\prime}} \delta_{m m^{\prime}} \delta_{s s^{\prime}} \frac{1}{\varepsilon_{m}},
\end{aligned}
$$

where

$$
\varepsilon_{m}= \begin{cases}1, & m=0 \\ 2, & m \geq 1\end{cases}
$$


Thus, for any $\mathbf{r} \in \Omega\left(\mathbb{R}^{3}\right)$, the internal vector spherical harmonics [7] are provided as

$$
\mathbf{N}_{n}^{(i) m s}(\mathbf{r})=\nabla\left(r^{n+1} Y_{n+1}^{m s}(\hat{\mathbf{r}})\right)=\sqrt{(n+1)(n+2)} r^{n} \mathbf{B}_{n+1}^{m s}(\hat{\mathbf{r}})+(n+1) r^{n} \mathbf{P}_{n+1}^{m s}(\hat{\mathbf{r}})
$$

for $n=0,1,2, \ldots, m=0,1, \ldots, n+1$, and $s=e, o$;

$$
\mathbf{M}_{n}^{(i) m s}(\mathbf{r})=\nabla \times\left(\mathbf{r} r^{n} Y_{n}^{m s}(\hat{\mathbf{r}})\right)=\sqrt{n(n+1)} r^{n} \mathbf{C}_{n}^{m s}(\hat{\mathbf{r}})
$$

for $n=1,2, \ldots, m=0,1, \ldots, n$, and $s=e, o$;

$$
\mathbf{G}_{n}^{(i) m s}(\mathbf{r})=r^{2 n+1} \mathbf{N}_{n}^{(e) m s}(\mathbf{r})=\sqrt{n(n-1)} r^{n} \mathbf{B}_{n-1}^{m s}(\hat{\mathbf{r}})-n r^{n} \mathbf{P}_{n-1}^{m s}(\hat{\mathbf{r}})
$$

for $n=0,1,2, \ldots, m=0,1, \ldots, n-1$, and $s=e, o$. On the other hand, the external vector spherical harmonics [7] assume the forms

$$
\mathbf{N}_{n}^{(e) m s}(\mathbf{r})=\nabla\left(r^{-n} Y_{n-1}^{m s}(\hat{\mathbf{r}})\right)=\sqrt{n(n-1)} r^{-(n+1)} \mathbf{B}_{n-1}^{m s}(\hat{\mathbf{r}})-n r^{-(n+1)} \mathbf{P}_{n-1}^{m s}(\hat{\mathbf{r}})
$$

for $n=1,2, \ldots, m=0,1, \ldots, n-1$, and $s=e, o$;

$$
\mathbf{M}_{n}^{(e) m s}(\mathbf{r})=\nabla \times\left(\mathbf{r} r^{-(n+1)} Y_{n}^{m s}(\hat{\mathbf{r}})\right)=\sqrt{n(n+1)} r^{-(n+1)} \mathbf{C}_{n}^{m s}(\hat{\mathbf{r}})
$$

for $n=1,2, \ldots, m=0,1, \ldots, n$, and $s=e, o$;

$$
\begin{aligned}
\mathbf{G}_{n}^{(e) m s}(\mathbf{r}) & =r^{-(2 n+1)} \mathbf{N}_{n}^{(i) m s}(\mathbf{r}) \\
& =\sqrt{(n+1)(n+2)} r^{-(n+1)} \mathbf{B}_{n+1}^{m s}(\hat{\mathbf{r}})+(n+1) r^{-(n+1)} \mathbf{P}_{n+1}^{m s}(\hat{\mathbf{r}})
\end{aligned}
$$

for $n=0,1,2, \ldots, m=0,1, \ldots, n+1$, and $s=e, o$. Then, the following complete expansion of any vector function $\mathbf{u}(\mathbf{r})$ which belongs to the kernel space of the operator $\Delta$ is obtained:

$$
\begin{aligned}
\mathbf{u}(\mathbf{r})= & \sum_{s=e, o} a_{0}^{(i) 0 s} \mathbf{N}_{0}^{(i) 0 s}(\mathbf{r})+\sum_{s=e, o} a_{0}^{(i) 1 s} \mathbf{N}_{0}^{(i) 1 s}(\mathbf{r}) \\
& +\sum_{s=e, o} c_{0}^{(e) 0 s} \mathbf{G}_{0}^{(e) 0 s}(\mathbf{r})+\sum_{s=e, o} c_{0}^{(e) 1 s} \mathbf{G}_{0}^{(e) 1 s}(\mathbf{r}) \\
& +\sum_{n=1}^{\infty} \sum_{m=0}^{n+1} \sum_{s=e, o} a_{n}^{(i) m s} \mathbf{N}_{n}^{(i) m s}(\mathbf{r})+\sum_{n=1}^{\infty} \sum_{m=0}^{n-1} \sum_{s=e, o} a_{n}^{(e) m s} \mathbf{N}_{n}^{(e) m s}(\mathbf{r}) \\
& +\sum_{n=1}^{\infty} \sum_{m=0}^{n} \sum_{s=e, o} b_{n}^{(i) m s} \mathbf{M}_{n}^{(i) m s}(\mathbf{r})+\sum_{n=1}^{\infty} \sum_{m=0}^{n} \sum_{s=e, o} b_{n}^{(e) m s} \mathbf{M}_{n}^{(e) m s}(\mathbf{r}) \\
& +\sum_{n=1}^{\infty} \sum_{m=0}^{n-1} \sum_{s=e, o} c_{n}^{(i) m s} \mathbf{G}_{n}^{(i) m s}(\mathbf{r})+\sum_{n=1}^{\infty} \sum_{m=0}^{n+1} \sum_{s=e, o} c_{n}^{(e) m s} \mathbf{G}_{n}^{(e) m s}(\mathbf{r})
\end{aligned}
$$

for every $\mathbf{r} \in \Omega\left(\mathbb{R}^{3}\right)$. In the interest of making this work more complete and independent, we provide in an appendix some relations between the vector spherical harmonics. The relevant information and recurrence relations for the associated Legendre functions of the first kind can be found in [4]. 


\section{PN eigenflows}

In view of equations (2.4), (2.5) and (3.9), (3.23), the harmonic eigenfunctions $\Phi(\mathbf{r})$ and $\Phi_{0}(\mathbf{r}), \mathbf{r} \in \Omega\left(\mathbb{R}^{3}\right)$, with constant coefficients $a_{n}^{(i) m s}, b_{n}^{(i) m s}, c_{n}^{(i) m s}, a_{n}^{(e) m s}, b_{n}^{(e) m s}, c_{n}^{(e) m s}$, and $d_{n}^{(i) m s}, d_{n}^{(e) m s}$, respectively,

$$
\begin{aligned}
\boldsymbol{\Phi}(\mathbf{r})= & \sum_{n=0}^{\infty} \sum_{m=0}^{n+1} \sum_{s=e, o} a_{n}^{(i) m s} \mathbf{N}_{n}^{(i) m s}(\mathbf{r})+\sum_{n=1}^{\infty} \sum_{m=0}^{n} \sum_{s=e, o} b_{n}^{(i) m s} \mathbf{M}_{n}^{(i) m s}(\mathbf{r}) \\
& +\sum_{n=1}^{\infty} \sum_{m=0}^{n-1} \sum_{s=e, o} c_{n}^{(i) m s} \mathbf{G}_{n}^{(i) m s}(\mathbf{r})+\sum_{n=1}^{\infty} \sum_{m=0}^{n-1} \sum_{s=e, o} a_{n}^{(e) m s} \mathbf{N}_{n}^{(e) m s}(\mathbf{r}) \\
& +\sum_{n=1}^{\infty} \sum_{m=0}^{n} \sum_{s=e, o} b_{n}^{(e) m s} \mathbf{M}_{n}^{(e) m s}(\mathbf{r})+\sum_{n=0}^{\infty} \sum_{m=0}^{n+1} \sum_{s=e, o} c_{n}^{(e) m s} \mathbf{G}_{n}^{(e) m s}(\mathbf{r}), \\
\Phi_{0}(\mathbf{r})= & \sum_{n=0}^{\infty} \sum_{m=0}^{n} \sum_{s=e, o} d_{n}^{(i) m s}\left(r^{n} Y_{n}^{m s}(\hat{\mathbf{r}})\right)+\sum_{n=0}^{\infty} \sum_{m=0}^{n} \sum_{s=e, o} d_{n}^{(e) m s}\left(r^{-(n+1)} Y_{n}^{m s}(\hat{\mathbf{r}})\right)
\end{aligned}
$$

generate the velocity and total pressure fields $\mathbf{v}^{\mathrm{PN}}, \mathrm{P}^{\mathrm{PN}}$. In terms of (3.11)-(3.22) and (A.1)-(A.11), the PN flow fields are written as

$$
\begin{aligned}
\mathbf{v}^{\mathrm{PN}}(\mathbf{r})= & \sum_{n=0}^{\infty} \sum_{m=0}^{n+1} \sum_{s=e, o}\left[-\frac{(n-1)}{2} a_{n}^{(i) m s}-\frac{1}{2} d_{n+1}^{(i) m s}+\frac{(n+2)(2 n+5)}{2(2 n+3)} c_{n+2}^{(i) m s} r^{2}\right] \mathbf{N}_{n}^{(i) m s}(\mathbf{r}) \\
& +\sum_{n=1}^{\infty} \sum_{m=0}^{n-1} \sum_{s=e, o}\left[\frac{(n+2)}{2} a_{n}^{(e) m s}-\frac{1}{2} d_{n-1}^{(e) m s}-\frac{(n-1)(2 n-3)}{2(2 n-1)} c_{n-2}^{(e) m s} r^{2}\right] \mathbf{N}_{n}^{(e) m s}(\mathbf{r}) \\
& +\sum_{n=1}^{\infty} \sum_{m=0}^{n} \sum_{s=e, o}\left[b_{n}^{(i) m s}\right] \mathbf{M}_{n}^{(i) m s}(\mathbf{r})+\sum_{n=1}^{\infty} \sum_{m=0}^{n} \sum_{s=e, o}\left[b_{n}^{(e) m s}\right] \mathbf{M}_{n}^{(e) m s}(\mathbf{r}) \\
& +\sum_{n=1}^{\infty} \sum_{m=0}^{n-1} \sum_{s=e, o}\left[\frac{(n-1)}{(2 n-1)} c_{n}^{(i) m s}\right] \mathbf{G}_{n}^{(i) m s}(\mathbf{r}) \\
& +\sum_{n=0}^{\infty} \sum_{m=0}^{n+1} \sum_{s=e, o}\left[\frac{(n+2)}{(2 n+3)} c_{n}^{(e) m s}\right] \mathbf{G}_{n}^{(e) m s}(\mathbf{r}), \\
& \left.+\sum_{n=0}^{\infty} \sum_{m=0}^{n} \sum_{s=e, o} n(2 n-1) c_{n-1}^{(e) m s}\left(r^{-(n+1)} Y_{n}^{m s}(\hat{\mathbf{r}})\right)\right\} \\
\mathrm{P}^{\mathrm{PN}}(\mathbf{r})= & \mathrm{P}_{0}^{\mathrm{PN}}+\mu\left\{\sum_{n=0}^{\infty} \sum_{m=0}^{n} \sum_{s=e, o}(n+1)(2 n+3) c_{n+1}^{(i) m s}\left(r^{n} Y_{n}^{m s}(\hat{\mathbf{r}})\right)\right. \\
&
\end{aligned}
$$

for every $\mathbf{r} \in \Omega\left(\mathbb{R}^{3}\right)$. 


\section{PNAU eigenflows}

According to (2.6), (2.7) and (3.9), (3.10), (3.23), the biharmonic and harmonic eigenfunctions $\mathrm{A}(\mathbf{r})$ and $\mathrm{B}(\mathbf{r}), \mathbf{r} \in \Omega\left(\mathbb{R}^{3}\right)$, with constant coefficients $f_{n}^{(i) m s}, g_{n}^{(i) m s}, f_{n}^{(e) m s}, g_{n}^{(e) m s}$, and $e_{n}^{(i) m s}, e_{n}^{(e) m s}$, respectively,

$$
\begin{aligned}
\mathrm{A}(\mathbf{r})= & \sum_{n=0}^{\infty} \sum_{m=0}^{n} \sum_{s=e, o} f_{n}^{(i) m s}\left(r^{n} Y_{n}^{m s}(\hat{\mathbf{r}})\right)+\sum_{n=0}^{\infty} \sum_{m=0}^{n} \sum_{s=e, o} f_{n}^{(e) m s}\left(r^{-(n+1)} Y_{n}^{m s}(\hat{\mathbf{r}})\right) \\
& +r^{2} \sum_{n=0}^{\infty} \sum_{m=0}^{n} \sum_{s=e, o} g_{n}^{(i) m s}\left(r^{n} Y_{n}^{m s}(\hat{\mathbf{r}})\right)+r^{2} \sum_{n=0}^{\infty} \sum_{m=0}^{n} \sum_{s=e, o} g_{n}^{(e) m s}\left(r^{-(n+1)} Y_{n}^{m s}(\hat{\mathbf{r}})\right), \\
\mathrm{B}(\mathbf{r})= & \sum_{n=0}^{\infty} \sum_{m=0}^{n} \sum_{s=e, o} e_{n}^{(i) m s}\left(r^{n} Y_{n}^{m s}(\hat{\mathbf{r}})\right)+\sum_{n=0}^{\infty} \sum_{m=0}^{n} \sum_{s=e, o} e_{n}^{(e) m s}\left(r^{-(n+1)} Y_{n}^{m s}(\hat{\mathbf{r}})\right)
\end{aligned}
$$

generate the PNAU velocity and total pressure fields $\mathbf{v}^{\mathrm{PNAU}}$ and $\mathrm{P}^{\mathrm{PNAU}}$ by virtue of (3.11)(3.22) as well as (A.1)-(A.11). That is,

$$
\begin{aligned}
\mathbf{v}^{\mathrm{PNAU}}(\mathbf{r})= & \sum_{n=0}^{\infty} \sum_{m=0}^{n+1} \sum_{s=e, o}\left[(n+2) f_{n+1}^{(i) m s}+\frac{(n+2)(2 n+5)}{(2 n+3)} g_{n+1}^{(i) m s} r^{2}\right] \mathbf{N}_{n}^{(i) m s}(\mathbf{r}) \\
& +\sum_{n=1}^{\infty} \sum_{m=0}^{n-1} \sum_{s=e, o}\left[-(n-1) f_{n-1}^{(e) m s}-\frac{(n-1)(2 n-3)}{(2 n-1)} g_{n-1}^{(e) m s} r^{2}\right] \mathbf{N}_{n}^{(e) m s}(\mathbf{r}) \\
& +\sum_{n=1}^{\infty} \sum_{m=0}^{n} \sum_{s=e, o}\left[e_{n}^{(i) m s}\right] \mathbf{M}_{n}^{(i) m s}(\mathbf{r})+\sum_{n=1}^{\infty} \sum_{m=0}^{n} \sum_{s=e, o}\left[e_{n}^{(e) m s}\right] \mathbf{M}_{n}^{(e) m s}(\mathbf{r}) \\
& +\sum_{n=1}^{\infty} \sum_{m=0}^{n-1} \sum_{s=e, o}\left[\frac{2(n-1)}{(2 n-1)} g_{n-1}^{(i) m s}\right] \mathbf{G}_{n}^{(i) m s}(\mathbf{r}) \\
& +\sum_{n=0}^{\infty} \sum_{m=0}^{n+1} \sum_{s=e, o}\left[\frac{2(n+2)}{(2 n+3)} g_{n+1}^{(e) m s}\right] \mathbf{G}_{n}^{(e) m s}(\mathbf{r}), \\
\mathrm{P}^{\mathrm{PNAU}}(\mathbf{r})= & \mathrm{P}_{0}^{\mathrm{PNAU}}+\mu\left\{\sum_{n=0}^{\infty} \sum_{m=0}^{n} \sum_{s=e, o} 2(n+1)(2 n+3) g_{n}^{(i) m s}\left(r^{n} Y_{n}^{m s}(\hat{\mathbf{r}})\right)\right. \\
& \left.+\sum_{n=0}^{\infty} \sum_{m=0}^{n} \sum_{s=e, o} 2 n(2 n-1) g_{n}^{(e) m s}\left(r^{-(n+1)} Y_{n}^{m s}(\hat{\mathbf{r}})\right)\right\}
\end{aligned}
$$

for every $\mathbf{r} \in \Omega\left(\mathbb{R}^{3}\right)$.

\section{Comparison of the PN and PNAU representations}

In this section, our aim is to find the exact harmonic and biharmonic potentials given by equations (4.1), (4.2) and (5.1), (5.2), which lead to the same velocity and total pressure fields. From this point of view, we look for connection formulae for the differential 
representations that secure the identities

$$
\mathbf{v}^{\mathrm{PN}}(\mathbf{r})=\mathbf{v}^{\mathrm{PNAU}}(\mathbf{r}), \quad \mathrm{P}^{\mathrm{PN}}(\mathbf{r})=\mathrm{P}^{\mathrm{PNAU}}(\mathbf{r}), \quad \mathbf{r} \in \Omega\left(\mathbb{R}^{3}\right)
$$

By virtue of (6.1), we proceed by interrelating the PN flow fields (4.3), (4.4) with the corresponding PNAU flow fields (5.3), (5.4). This correlation leads to connection formulae that interrelate the corresponding constant coefficients of the potentials (4.1), (4.2), (5.1), and (5.2). What is actually happening is that the connection of the velocity and total pressure fields has been transferred to the corresponding connection of the constant coefficients of the potentials. Indeed, after some calculations, we obtain the relations

$$
\begin{gathered}
c_{n+1}^{(i) m s}=2 g_{n}^{(i) m s} \quad \text { for } n=0,1,2, \ldots, m=0,1, \ldots, n, s=e, o, \\
c_{n-1}^{(e) m s}=2 g_{n}^{(e) m s} \quad \text { for } n=1,2, \ldots, m=0,1, \ldots, n, s=e, o, \\
b_{n}^{(i) m s}=e_{n}^{(i) m s} \text { for } n=1,2, \ldots, m=0,1, \ldots, n, s=e, o, \\
b_{n}^{(e) m s}=e_{n}^{(e) m s} \text { for } n=1,2, \ldots, m=0,1, \ldots, n, s=e, o, \\
(n-2) a_{n-1}^{(i) m s}+d_{n}^{(i) m s}=-2(n+1) f_{n}^{(i) m s} \quad \text { for } n=1,2, \ldots, m=0,1, \ldots, n, s=e, o, \\
(n+3) a_{n+1}^{(e) m s}-d_{n}^{(e) m s}=-2 n f_{n}^{(e) m s} \quad \text { for } n=1,2, \ldots, m=0,1, \ldots, n, s=e, o,
\end{gathered}
$$

which establish the connection between the PN and PNAU representations at the coefficient level. The cases that do not follow the general relations (6.2)-(6.7) for $n=0$ are treated separately. These concern the coefficients

$$
g_{0}^{(e) 0 e}, e_{0}^{(i) 0 e}, e_{0}^{(e) 0 e}, d_{0}^{(i) 0 e}, f_{0}^{(i) 0 e}, f_{0}^{(e) 0 e} \in \mathbb{R}
$$

Furthermore, the interrelation of the total pressures implies the equation of the constant pressures defined earlier, that is,

$$
\mathrm{P}_{0}^{\mathrm{PN}}=\mathrm{P}_{0}^{\mathrm{PNAU}}
$$

Flows of zero vorticity are irrotational flows. Consequently, irrotational fields force the corresponding terms of the potentials, or of the flow fields, to vanish. Then, according to (4.3) and (5.3) of the velocity fields, in view of (2.3) and the relations (A.7), (A.9), and (A.11), the following constant coefficients are set to zero on the basis of orthogonality arguments:

$$
\begin{array}{ll}
c_{n+1}^{(i) m s}=g_{n}^{(i) m s}=0 & \text { for } n=0,1,2, \ldots, m=0,1, \ldots, n, s=e, o, \\
c_{n-1}^{(e) m s}=g_{n}^{(e) m s}=0 & \text { for } n=1,2, \ldots, m=0,1, \ldots, n, s=e, o, \\
b_{n}^{(i) m s}=e_{n}^{(i) m s}=0 & \text { for } n=1,2, \ldots, m=0,1, \ldots, n, s=e, o, \\
b_{n}^{(e) m s}=e_{n}^{(e) m s}=0 & \text { for } n=1,2, \ldots, m=0,1, \ldots, n, s=e, o .
\end{array}
$$


Even though the biharmonic part of the biharmonic potential A and the harmonic potential B are connected directly to the G-component and the $\mathbf{M}$-component of the harmonic potential $\Phi$, respectively, as shown from the general equations (6.2)-(6.5), the procedure of interrelation is not invertible. The reason for this lack of invertibility is due to the general connection relations (6.6) and (6.7), where the harmonic part of the biharmonic potential A is given through the $\mathrm{N}$-component of the harmonic potential $\Phi$ and through the harmonic potential $\Phi_{0}$. The transformation from one representation to the other is not obtainable analytically in the sense that one can start with the PN differential representation and regain the results from the PNAU differential solution through the relations above, but one cannot come the opposite way since two sets of internal and external constant coefficients of the PN solution cannot be determined. Consequently, we deal with a higher number of degrees of freedom for the PN differential representation, a fact that implies the flexibility of the PN representation. In other words, for the same eigenflow, the PN representation lives in a higher-dimensional space than the PNAU one.

\section{Application: the Kuwabara sphere-in-cell model}

In order to demonstrate the usefulness of the PN differential representation $((2.4),(2.5)$ or $(4.3),(4.4))$, we use it to solve the axisymmetric Stokes flow problem through a swarm of stationary spherical particles, embedded within an otherwise quiescent Newtonian fluid that moves with constant uniform velocity in the polar direction. In other words, according to the idea of particle-in-cell models described in the introduction, we are interested in solving the creeping flow within a fluid cell limited between two concentric spherical surfaces.

Two concentric spheres are considered. The inner one, indicated by $S_{\alpha}$, at $r=\alpha$, is solid and stationary. It lives within a spherical layer, which is confined by the outer sphere indicated by $S_{b}$, at $r=b$. A uniformly approaching velocity of magnitude $U$, in the negative direction of the $x_{3}$-axis, generates the axisymmetric flow in the fluid layer between the two spheres. The boundary conditions assume the forms

$$
\begin{aligned}
v_{r} & =0 \quad \text { on } r=\alpha, \\
v_{\zeta} & =0 \quad \text { on } r=\alpha, \\
v_{r} & =-U \zeta \quad \text { on } r=b, \\
\omega_{\varphi} & \equiv \hat{\boldsymbol{\varphi}} \cdot \boldsymbol{\omega}=0 \quad \text { on } r=b,
\end{aligned}
$$

where $v_{r}$ and $v_{\zeta}$ are the $r$ and $\zeta$ components of the axisymmetric PN velocity field and $\omega_{\varphi}$ refers to the $\varphi$ component of the vorticity field given by (2.3). Equations (7.1) and (7.2) express the nonslip flow condition. Equation (7.3) implies that there is a flow across the boundary of the fluid envelope $S_{b}$. Furthermore, according to the Kuwabara argument, the vorticity is assumed to vanish on the external sphere, as shown by equation (7.4). This completes the statement of a well-posed boundary value problem.

Since the PN representation covers 3D flow fields, for 2D flows, as in our case, we are obliged to make a considerable reduction considering rotational symmetry. This is attainable and requires the same velocity field on every meridian plane. That is, the velocity is 
independent of the azimuthal angle $\varphi$ :

$$
\partial \mathbf{v}^{\mathrm{PN}}(\mathbf{r}) \partial \varphi=\mathbf{0}, \quad \mathbf{r} \in \Omega\left(\mathbb{R}^{3}\right)
$$

and its vector lives on a meridian plane:

$$
\hat{\boldsymbol{\varphi}} \cdot \mathbf{v}^{\mathrm{PN}}(\mathbf{r})=0, \quad \mathbf{r} \in \Omega\left(\mathbb{R}^{3}\right) .
$$

Now, imposing the axisymetric conditions (7.5) and (7.6) to our representation, the velocity field (4.3) is written in a suitable form:

$$
\mathbf{v}^{\mathrm{PN}}(r, \zeta)=v_{r}^{\mathrm{PN}}(r, \zeta) \hat{\mathbf{r}}+v_{\zeta}^{\mathrm{PN}}(r, \zeta) \hat{\zeta}, \quad r>0,|\zeta| \leq 1,
$$

where the components of the velocity are expressed in terms of the radial component and Legendre functions of the first kind via

$$
\begin{aligned}
v_{r}^{\mathrm{PN}}(r, \zeta)=\sum_{n=0}^{\infty} \frac{1}{2}\{ & (n+1)\left(\frac{(n+3)}{(2 n+3)} \tilde{c}_{n+1}^{(e)}+\tilde{d}_{n}^{(e)}\right) r^{-(n+2)}+\frac{n(n+1)}{(2 n-1)} \tilde{c}_{n-1}^{(e)} r^{-n} \\
& \left.-n\left(\frac{(n-2)}{(2 n-1)} \tilde{c}_{n-1}^{(i)}+\tilde{d}_{n}^{(i)}\right) r^{n-1}-\frac{n(n+1)}{(2 n+3)} \tilde{c}_{n+1}^{(i)} r^{n+1}\right\} P_{n}(\zeta), \\
v_{\zeta}^{\mathrm{PN}}(r, \zeta)=\sum_{n=1}^{\infty} \frac{1}{2}\{ & \left(\frac{(n+3)}{(2 n+3)} \tilde{c}_{n+1}^{(e)}+\tilde{d}_{n}^{(e)}\right) r^{-(n+2)}+\frac{(n-2)}{(2 n-1)} \tilde{c}_{n-1}^{(e)} r^{-n} \\
+ & \left.\left(\frac{(n-2)}{(2 n-1)} \tilde{c}_{n-1}^{(i)}+\tilde{d}_{n}^{(i)}\right) r^{n-1}+\frac{(n+3)}{(2 n+3)} \tilde{c}_{n+1}^{(i)} r^{n+1}\right\} P_{n}^{1}(\zeta),
\end{aligned}
$$

while for the total pressure we obtain, from equation (4.4),

$$
\mathrm{P}^{\mathrm{PN}}(r, \zeta)=\mathrm{P}_{0}^{\mathrm{PN}}-\mu \sum_{n=0}^{\infty}\left\{(n+1) \tilde{c}_{n+1}^{(i)} r^{n}-n \tilde{c}_{n-1}^{(e)} r^{-(n+1)}\right\} P_{n}(\zeta), \quad r>0,|\zeta| \leq 1
$$

The vorticity field given in (2.3), in view of (7.7), (7.8), and (7.9), is easily confirmed to be expressible in

$$
\boldsymbol{\omega}^{\mathrm{PN}}(r, \zeta)=\hat{\varphi} \omega_{\varphi}^{\mathrm{PN}}(r, \zeta), \quad r>0,|\zeta| \leq 1
$$

whereas

$$
\omega_{\varphi}^{\mathrm{PN}}(r, \zeta)=\sum_{n=1}^{\infty}\left\{\tilde{c}_{n+1}^{(i)} r^{n}+\tilde{c}_{n-1}^{(e)} r^{-(n+1)}\right\} P_{n}^{1}(\zeta)
$$

The constant coefficients $\tilde{c}_{n}^{(i)}, \tilde{c}_{n}^{(e)}, \tilde{d}_{n}^{(i)}$, and $\tilde{d}_{n}^{(e)}, n \geq 0$, must be determined from the appropriate boundary conditions. 
In order to apply the boundary conditions (7.1)-(7.4), we use the expressions (7.8), (7.9), and (7.12) as well as certain recurrence and orthogonality relations for the Legendre functions [4]. After some extensive algebra, one obtains a complicated system of linear algebraic equations involving the unknown constant coefficients, where only the first term provides us with the solution and then we obtain the corrected solution of the Kuwabara-type boundary value problem [5], that is,

$$
\begin{aligned}
& \mathbf{v}^{\mathrm{PN}}(r, \zeta)=v_{r}^{\mathrm{PN}}(r, \zeta) \hat{\mathbf{r}}+v_{\zeta}^{\mathrm{PN}}(r, \zeta) \hat{\zeta} \\
& v_{r}^{\mathrm{PN}}(r, \zeta)=\frac{U \zeta}{2 K}\left[\frac{3}{5 \ell^{3}}\left(\frac{r}{\alpha}\right)^{2}-\left(2+\frac{1}{\ell^{3}}\right)+3\left(\frac{\alpha}{r}\right)-\left(1-\frac{2}{5 \ell^{3}}\right)\left(\frac{\alpha}{r}\right)^{3}\right] \\
& v_{\zeta}^{\mathrm{PN}}(r, \zeta)=-\frac{U \sqrt{1-\zeta^{2}}}{2 K}\left[\frac{6}{5 \ell^{3}}\left(\frac{r}{\alpha}\right)^{2}-\left(2+\frac{1}{\ell^{3}}\right)+\frac{3}{2}\left(\frac{\alpha}{r}\right)+\frac{1}{2}\left(1-\frac{2}{5 \ell^{3}}\right)\left(\frac{\alpha}{r}\right)^{3}\right], \\
& \boldsymbol{\omega}^{\mathrm{PN}}(r, \zeta)=\hat{\boldsymbol{\varphi}} \frac{3 U \sqrt{1-\zeta^{2}}}{2 \alpha K}\left[-\frac{1}{\ell^{3}}\left(\frac{r}{\alpha}\right)+\left(\frac{\alpha}{r}\right)^{2}\right] \\
& \mathrm{P}^{\mathrm{PN}}(r, \zeta)=\mathrm{P}_{0}^{\mathrm{PN}}+\frac{3 \mu U \zeta}{2 \alpha K}\left[\frac{2}{\ell^{3}}\left(\frac{r}{\alpha}\right)+\left(\frac{\alpha}{r}\right)^{2}\right]
\end{aligned}
$$

where $\ell=b / \alpha>1, K=(\ell-1)^{3}\left(1+3 \ell+6 \ell^{2}+5 \ell^{3}\right) / 5 \ell^{6}$, and $\alpha, b$ are the radii of the concentric spheres. We remark here on the simple way one can obtain the solution preserving at the same time the mathematical rigor.

\section{Conclusions}

A method for connecting two differential representations for nonaxisymmetric Stokes flow was developed. Based on this method, we examined the Papkovich-Neuber (PN) $[8,10]$ and the Palaniappan et al. (PNAU) [9] differential representations, which offer solutions for such flow problems in spherical geometry. The important physical flow fields (velocity, total pressure) are presented in terms of vector spherical harmonics. Furthermore, interrelation of the flow fields leads to connection formulae for the constant coefficients of the potentials, using the corresponding potentials as a function of spherical eigenfunctions. An immediate consequence of the interrelation of our representations for Stokes flow is that this procedure cannot be inverted. Consequently, one can always calculate the flow fields via the PNAU representation once the PN eigenmodes are known, but one cannot obtain relations that provide the PN potentials through the harmonic and biharmonic PNAU potentials.

An application of the present theory to an axisymmetric Stokes flow problem in a spherical cell (as a mean of modeling flow through a swarm of spherical particles) with the help of the PN differential representation was provided. An extension of the problem presented here to the case of ellipsoidal geometry for the creeping flow of small ellipsoidal particles is under current investigation. 


\section{Appendix}

For completeness, we present the following relations between the vector surface and solid spherical harmonics:

$$
\begin{aligned}
\mathbf{B}_{n}^{m s} \times \mathbf{P}_{n}^{m s} & =Y_{n}^{m s} \mathbf{C}_{n}^{m s}, \quad \mathbf{P}_{n}^{m s} \times \mathbf{C}_{n}^{m s}=Y_{n}^{m s} \mathbf{B}_{n}^{m s}, \\
\mathbf{B}_{n}^{m s} & =\hat{\mathbf{r}} \times \mathbf{C}_{n}^{m s}, \quad \mathbf{C}_{n}^{m s}=\mathbf{B}_{n}^{m s} \times \hat{\mathbf{r}}, \\
\mathbf{P}_{n}^{m s}(\hat{r}) & =\frac{r^{-n+1}}{(2 n+1)} \mathbf{N}_{n-1}^{(i) m s}(\mathbf{r})-\frac{r^{n+2}}{(2 n+1)} \mathbf{N}_{n+1}^{(e) m s}(\mathbf{r}), \\
\mathbf{B}_{n}^{m s}(\hat{\mathbf{r}}) & =\sqrt{\frac{n+1}{n}} \frac{r^{-n+1}}{(2 n+1)} \mathbf{N}_{n-1}^{(i) m s}(\mathbf{r})+\sqrt{\frac{n}{n+1}} \frac{r^{n+2}}{(2 n+1)} \mathbf{N}_{n+1}^{(e) m s}(\mathbf{r}), \\
\mathbf{C}_{n}^{m s}(\hat{\mathbf{r}}) & =\frac{r^{-n}}{2 \sqrt{n(n+1)}} \mathbf{M}_{n}^{(i) m s}(\mathbf{r})+\frac{r^{n+1}}{2 \sqrt{n(n+1)}} \mathbf{M}_{n}^{(e) m s}(\mathbf{r}),
\end{aligned}
$$

for $n=0,1,2, \ldots, m=0,1, \ldots, n+1, s=e, o$, and $\mathbf{r} \in \Omega\left(\mathbb{R}^{3}\right)$. Finally, for the vector spherical harmonics, one can easily derive the following relations:

$$
\begin{aligned}
& \nabla \cdot \mathbf{N}_{n}^{(i) m s}(\mathbf{r})=0, \quad \nabla \cdot \mathbf{N}_{n}^{(e) m s}(\mathbf{r})=0, \\
& \nabla \times \mathbf{N}_{n}^{(i) m s}(\mathbf{r})=\mathbf{0}, \quad \nabla \times \mathbf{N}_{n}^{(e) m s}(\mathbf{r})=\mathbf{0}, \\
& \nabla \cdot \mathbf{M}_{n}^{(i) m s}(\mathbf{r})=0, \quad \nabla \cdot \mathbf{M}_{n}^{(e) m s}(\mathbf{r})=0, \\
& \nabla \times \mathbf{M}_{n}^{(i) m s}(\mathbf{r})=(n+1) \mathbf{N}_{n-1}^{(i) m s}(\mathbf{r}), \quad \nabla \times \mathbf{M}_{n}^{(e) m s}(\mathbf{r})=-n \mathbf{N}_{n+1}^{(e) m s}(\mathbf{r}), \\
& \nabla \cdot \mathbf{G}_{n}^{(i) m s}(\mathbf{r})=-n(2 n+1) r^{n-1} Y_{n-1}^{m s}(\hat{\mathbf{r}}), \\
& \nabla \cdot \mathbf{G}_{n}^{(e) m s}(\mathbf{r})=-(n+1)(2 n+1) r^{-(n+2)} Y_{n+1}^{m s}(\hat{\mathbf{r}}), \\
& \nabla \times \mathbf{G}_{n}^{(i) m s}(\mathbf{r})=-(2 n+1) \mathbf{M}_{n-1}^{(i) m s}(\mathbf{r}), \quad \nabla \times \mathbf{G}_{n}^{(e) m s}(\mathbf{r})=(2 n+1) \mathbf{M}_{n+1}^{(e) m s}(\mathbf{r}) .
\end{aligned}
$$

\section{References}

[1] M. J. Boussinesq, Application des Potentiels a l' Étude de l' Équilibre et du Mouvement des Solides Élastiques, Gauthier-Villars, Paris, 1885.

[2] G. Dassios and P. Vafeas, Connection formulae for differential representations in Stokes flow, J. Comput. Appl. Math. 133 (2001), no. 1-2, 283-294.

[3] R. W. Fox and A. T. McDonald, Introduction to Fluid Mechanics, 3rd ed., John Wiley \& Sons, New York, 1985.

[4] E. W. Hobson, The Theory of Spherical and Ellipsoidal Harmonics, Chelsea, New York, 1955.

[5] S. Kuwabara, The forces experienced by randomly distributed parallel circular cylinders or spheres in a viscous flow at small Reynolds numbers, J. Phys. Soc. Japan 14 (1959), 527-532.

[6] P. Moon and E. Spencer, Partial Differential Equations, Heath \& Company, Massachusetts, 1969.

[7] P. M. Morse and H. Feshbach, Methods of Theoretical Physics Vols I, II, McGraw-Hill, New York, 1953.

[8] H. Neuber, Ein neuer Ansatz zur Lösung räumlicher Probleme der Elastizitätstheorie, Z. Angew. Math. Mech. 14 (1934), 203-212 (German). 
[9] D. Palaniappan, S. D. Nigam, T. Amaranath, and R. Usha, Lamb's solution of Stokes's equations: a sphere theorem, Quart. J. Mech. Appl. Math. 45 (1992), no. 1, 47-56.

[10] X. Xu and M. Wang, General complete solutions of the equations of spatial and axisymmetric Stokes flow, Quart. J. Mech. Appl. Math. 44 (1991), no. 4, 537-548.

George Dassios: Division of Applied Mathematics, Department of Chemical Engineering, University of Patras, 26504 Patras; Institute of Chemical Engineering and High Temperature Chemical Processes (ICE/HT), Foundation for Research Technology-Hellas (FORTH), 26504 Patras, Greece

E-mail address: dassios@chemeng.upatras.gr

Panayiotis Vafeas: Division of Applied Mathematics, Department of Chemical Engineering, University of Patras, 26504 Patras; Institute of Chemical Engineering and High Temperature Chemical Processes (ICE/HT), Foundation for Research Technology-Hellas (FORTH), 26504 Patras, Greece

E-mail address: vafeas@chemeng.upatras.gr 


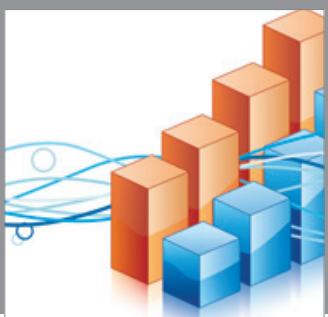

Advances in

Operations Research

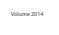

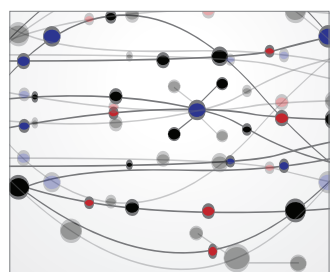

\section{The Scientific} World Journal
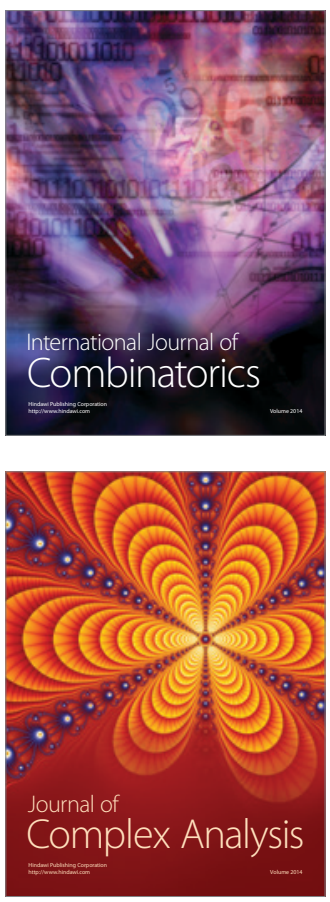

International Journal of

Mathematics and

Mathematical

Sciences
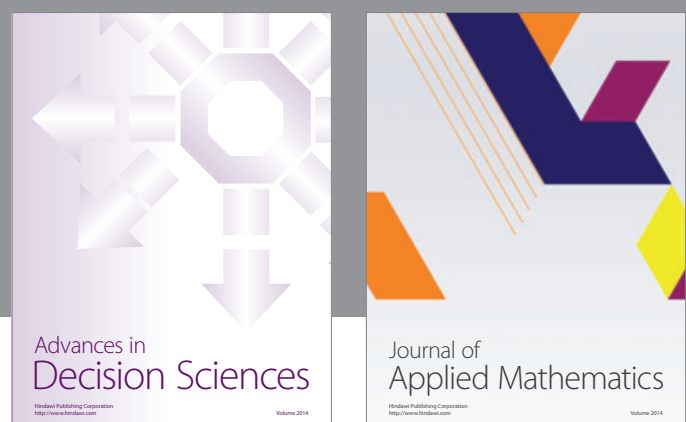

Journal of

Applied Mathematics
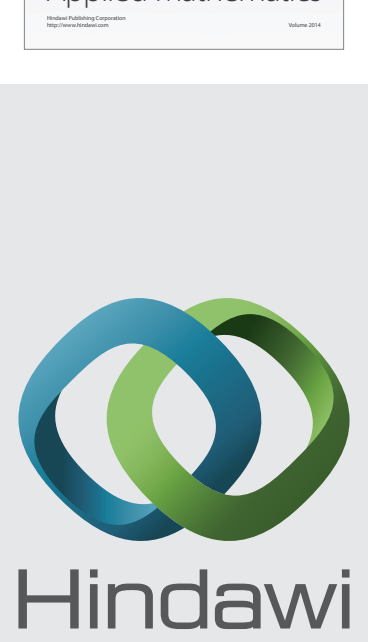

Submit your manuscripts at http://www.hindawi.com
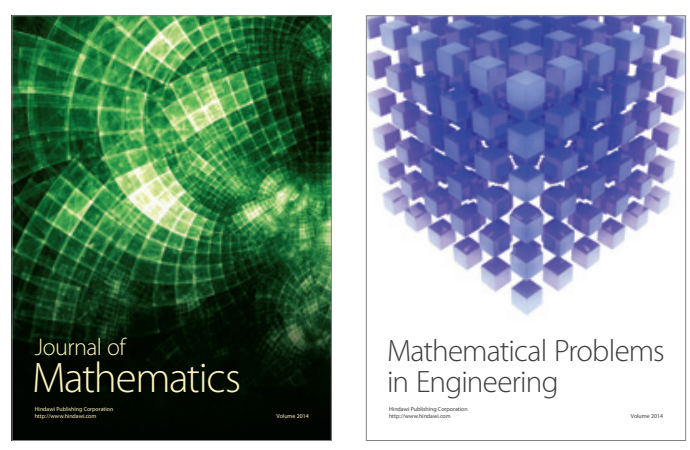

Mathematical Problems in Engineering
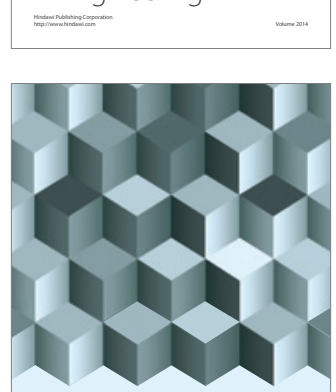

Journal of

Function Spaces
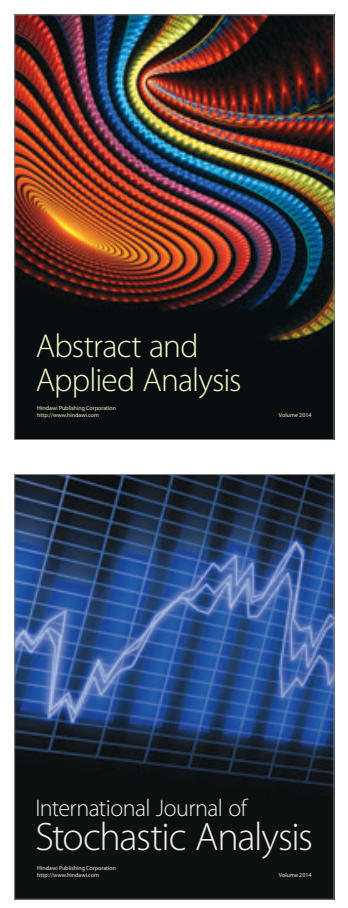

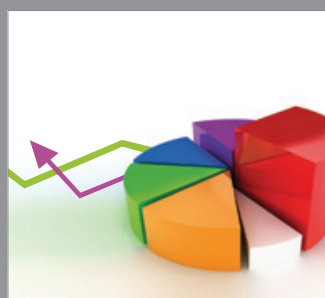

ournal of

Probability and Statistics

Promensencen
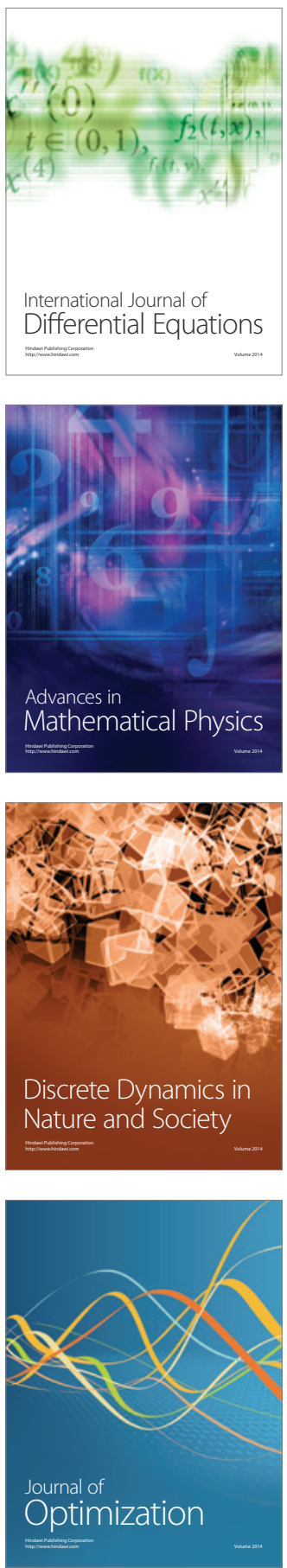